\title{
Publisher's Note: Spin Textures of Exciton-Polaritons in a Tunable Microcavity with Large TE-TM Splitting [Phys. Rev. Lett. 115, 246401 (2015)]
}

S. Dufferwiel, Feng Li, E. Cancellieri, L. Giriunas, A. A. P. Trichet, D. M. Whittaker, P. M. Walker,

F. Fras, E. Clarke, J. M. Smith, M. S. Skolnick, and D. N. Krizhanovskii

(Received 2 February 2017; published 22 February 2017)

DOI: 10.1103/PhysRevLett.118.089901

This Letter was published online on 8 December 2015 with missing information in the Acknowledgments. The Acknowledgments should read as "We acknowledge support by EPSRC Grant No. EP/J007544, ERC Advanced Grant EXCIPOL, and the Leverhulme Trust Grant No. RPG-2013-339." The Acknowledgments have been corrected as of 10 February 2017. The Acknowledgments are incorrect in the printed version of the journal.

Published by the American Physical Society under the terms of the Creative Commons Attribution 4.0 International license. Further distribution of this work must maintain attribution to the author(s) and the published articles title, journal citation, and DOI. 